\title{
Personalised cancer care in the era of precision medicine
}

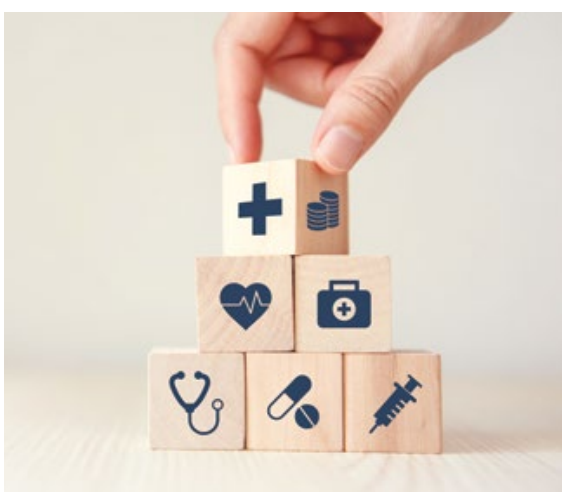

CPD

Bogda Koczwara, Elysia Thornton-Benko, Richard J Cohn, Raymond J Chan, Joel Rhee, David Joske, Mahesh Iddawela, Janette L Vardy

\section{Background}

Advances in cancer treatment have not benefited all patients equally, underscoring the need for a personalised approach to care.

\section{Objective}

The aim of this article is to outline the key elements of personalised cancer care, including delivery of goal-directed care, self-management and selfmanagement support, care integration, focus on access and equity, reduction in cost and promotion of health literacy and e-health literacy.

\section{Discussion}

Achievement of personalised cancer care requires a system-wide approach that targets the patient, healthcare provider and healthcare system with data informing practice. Primary care providers, including general practitioners (GPs) and practice nurses, play an important and growing part in the provision of personalised cancer care through support, advocacy, coordination, holistic care and health promotion. Cancer care systems can facilitate GPs' involvement in care through early input into multidisciplinary management, timely communication, rapid access to acute care and training opportunities.
THE BEGINNING OF THE 21ST CENTURY has seen unprecedented advances in cancer control, ${ }^{1}$ including refinement of existing treatments, introduction of new therapies and advances in molecular diagnostics to enable the selection of therapy to match the unique characteristics of the tumour, commonly described as precision medicine. ${ }^{2}$ These advances have led to significant improvement in cure rates and extended survival for those whose cancer cannot be cured. For some cancers, such as chronic myeloid leukemia, the cancer-free survival approximates that of the cancer-free population. ${ }^{3}$

However, these significant advances have not benefited all patients with cancer equally, with variability in outcomes relating to access, comorbidities and other factors. This highlights the importance of tailoring care to individual patients' circumstances to deliver not only precision therapeutics, but also personalised, patient-centred care. ${ }^{4}$ In this article, the authors outline the key elements of personalised cancer care, with a particular focus on the Australian context.

\section{Personalised care}

While it is accepted that healthcare should be safe and effective, quality healthcare is also expected to be person-centred (acceptable to the patient), accessible, efficient (integrated) and equitable. ${ }^{5}$
Patient-centred care has been defined as 'respectful of and responsive to individual preferences, needs and values' ${ }^{6}$ These preferences and needs inform the goals of care and the engagement in care at the level acceptable to the patient.

To respond to patients' needs and preferences, healthcare providers need to regularly assess patients' needs, preferably using a standardised approach with patient-reported outcome measures (PROMs). There is now high-level evidence that PROMs collection in oncology is associated with improved patient satisfaction, a reduction in emergency presentations and facilitation of shared decision making. ${ }^{7}$ There are now ongoing, albeit slow, efforts to implement PROMs in specialist cancer care clinics. Further cross-sectorial information and communications technology integration will likely see an increasing relevance of PROMs to primary care providers.

\section{Goal-directed care}

The concept of goals of treatment (palliative versus curative) has been a core concept in oncology since its inception; however, as with precision therapeutics, its origins focus on the cancer rather than the person with cancer and may not accurately reflect the implications of cancer treatment. For example, many cancers that are not cured can be effectively controlled 
by anticancer treatment for extended periods. Conversely, in some patients who are free from cancer after curative treatment, their life expectancy may be shortened because of late side effects of cancer treatment. The goals of care need to take into account patients' goals. These may include a wish to travel, to attend a family event or to be well enough to continue working. Eliciting goals and priorities is a standard component of chronic disease management and care plan development, but these approaches are yet to be routinely adopted in oncology. ${ }^{8}$ As such, primary care providers have an important role in advocating for patients' goals within the wider multidisciplinary team.

\section{Self-management and self-management support}

This goal-directed approach includes clarification of patients' preferences regarding how they wish to engage with the healthcare process and what role they can play through self-management. ${ }^{9}$ Self-management is defined as 'the individual's ability to manage the symptoms, treatment, physical and psychosocial consequences and lifestyle changes inherent in living with a chronic condition' ${ }^{10}$ The rapidly changing cancer care environment, with many aspects of care being shifted to the home setting, has created a pressing need for patients and informal/family caregivers to self-monitor and self-manage impacts of their cancer, treatment-related side effects and cancerrelated symptoms in the context of their overall health. General practitioners (GPs) and practice nurses, in collaboration with the multidisciplinary healthcare team, have pivotal roles in providing self-management support and improving patient outcomes throughout the cancer care trajectory. ${ }^{11-13}$ GPs and practice nurses can apply effective self-management support strategies for patients during primary care consultations. ${ }^{10}$ These strategies may include actions such as improving patients' disease and treatment knowledge, facilitating self-monitoring of symptoms, encouraging self-treatment through a personalised action plan in response to symptoms or health concerns, suggesting coping and stress management strategies, and empowering patients to make healthy lifestyle choices. Regular follow-up incorporating personalised feedback, monitoring and supporting progress related to healthcare goal setting, or honing problem-solving skills are key components of this self-management support. Peer support, where available and desirable by patients, may also be helpful in supporting effective self-management. ${ }^{14}$

\section{Care integration}

Cancer care is inherently complex, often requiring multiple treatment modalities including surgery, chemotherapy, radiation therapy and support services such as rehabilitation and reconstruction. Many patients have pre-existing comorbid conditions, with the severity and prevalence of comorbid conditions increasing after cancer diagnosis. ${ }^{15}$ Integrated care requires effective coordination to ensure continuity of care with integration of the patient's goals and clear communication between all team members and the patient. While some patients in Australia are supported by cancer care coordinators, many patients do not have access to a coordinator, and not every coordinator works within models that adopt a holistic whole-patient care approach.

Integration of care involves horizontal integration across cancer services and vertical integration of acute cancer treatment with primary care and secondary prevention and health promotion. This is particularly relevant to the care of cancer survivors where these aspects of care are essential for effective survivorship care. ${ }^{16}$ Integrated care is not only less fragmented, less costly and more efficient, but also safer and more effective as it minimises the possibility that treatment for one condition, recommended by one provider, interferes with the outcomes of another.

\section{Access to care}

Quality cancer care must be accessible. In Australia, access to care is frequently limited by distance, with patients living in rural and remote Australia having less access to specialty cancer services and to healthcare in general, which contributes to poorer cancer outcomes in rural Australia. ${ }^{17}$ The introduction of rural cancer services such as telemedicine and teletrials ${ }^{18}$ to many parts of Australia has increased access to acute cancer treatment, but supportive care, rehabilitation and secondary prevention integration is yet to be achieved. As GPs and practice nurses are the key healthcare providers in rural communities, they can play an important part in the delivery of these interventions and require appropriate support to do so through training, access to interventions and funding.

\section{Equitable care}

Access to care is not just a function of geography. Personalised cancer care needs to take into account that patients may have different abilities to engage with the healthcare system because of varying levels of social support, financial resources and health literacy. Cost of care and resulting 'financial toxicity' has been recently recognised as an important issue for patients and their families, magnified by difficulties in work participation. ${ }^{19}$ There is a need for a systematic approach to recognition, prevention and management of financial toxicity, and GPs can play an important part in this process.

Accessing support and navigating the healthcare system requires sufficient health literacy - a level of knowledge, personal skills and confidence to take action to improve health - and extends beyond the ability to read medical information. ${ }^{20}$ As with self-management, individual health literacy is supported by the appropriate health literacy environment. Increasingly, engagement with healthcare requires access to digital health (such as telehealth) and proficiency in e-health literacy. Yet, rates of digital inclusion in Australia vary significantly, with South Australia having the lowest rates, possibly because of an older population and large degree of remoteness. Digital inclusion relates not just to e-health literacy (ability to use) but accessibility of technology and its affordability. ${ }^{21}$ Without 
addressing the equitability of e-health in cancer care, future advances can accentuate gaps in care. ${ }^{22}$

\section{Achieving personalised cancer care: Next steps}

The path to personalised cancer care must ensure that care is patient-centred (goal-directed, enabling self-management and integrated), accessible and equitable. Adoption of these principles into care practices requires a focus not just on an individual healthcare provider and patient, but also on the health system as a whole.

Data are lacking to assist in service planning and research priorities, including mortality and morbidity data according to socioeconomic status, and comorbidities. Research should focus on disparities, comorbidities, digital inclusion and care integration from the perspectives of consumers, including the most vulnerable populations, and healthcare providers that support them. Once interventions are ready for adoption into clinical practice, there is a need for system-level levers such as embedding them into clinical workflows and reimbursement.

These broad system changes need to be adopted at the health service level, including the primary care setting (Box 1). GPs and practice nurses can play a critical part in facilitating care coordination, management of comorbidities and health promotion, and they are often well aware of unique needs of patients that go beyond their cancer diagnosis and extend to their families and caregivers. ${ }^{23}$ However, existing models of care are not well aligned with these goals. People with cancer have complex healthcare needs, and their care by primary care providers must be supported by clear practice guidelines, access to advice and referrals, resources and time for prolonged consultation with appropriate reimbursement. Patients who do not have a regular GP should be encouraged to find one. Oncology teams need to enquire about the role that the GP plays in the patient's care and support and value it. GPs input needs to be integrated into the overall management approach as part of the multidisciplinary care team, but these meetings traditionally do not involve the patient nor their GP, who could provide more context into their overall healthcare needs. Historically this lack of involvement was explained by the difficulties associated with attending in person, but this could be overcome by using telemedicine for GPs to connect. This multidisciplinary team interaction would further be optimised with timely, easy-to-access, multiway communication from all involved health professionals. For many cancer survivors, GPs can play a central part by providing a continuum of care (Box 2).

Lastly, this 'personalisation' of cancer care needs a broader change of mindset/ cultural change and language change so that personalisation of care is seen as just as relevant to cancer care as precision medicine and not an afterthought.

\section{Conclusion}

It is time to make cancer care not just technically sophisticated, but also personalised and holistic. Bringing together the precision and personalisation of cancer care offers a chance to improve the quality of cancer care for individual patients with cancer. In this way, the promise of precision oncology can be realised within the context of patients' goals and capabilities, with the support of the health system, leading to better patient outcomes and likely better health system performance.

\section{Key points}

- Primary care providers have an important and growing role in the provision of personalised cancer care through support, advocacy, coordination, holistic care and health promotion.

- Cancer care systems can facilitate GPs' involvement in care through early endorsement of GP input, involvement of GPs in multidisciplinary management, timely communication, rapid access to acute care and training opportunities.

\section{Authors}

Bogda Koczwara BMBS, MBioethics, Senior Staff Specialist, Department of Medical Oncology,

Flinders Medical Centre and Flinders University, Adelaide, SA

\section{Box 1. Taking action to enable personalised care: What can general practitioners do?}

General practitioners (GPs) and primary care providers have a crucial and growing role in delivery of cancer care, including:

- patient support and education

- advocacy for patients and caregivers

- help with care navigation and coordination

- provision of holistic general care

- management of comorbidities

- management of short- and long-term treatment toxicities

- supporting patient self-management

- health promotion

- cancer surveillance

- caregiver support.

Health systems can support GPs in their role by:

- acknowledging and reinforcing the role of the GP as part of the overall care team

- ensuring correct contact details for the cancer service and the GP are available to all providers and the patient

- early involvement in multidisciplinary decision making and care planning

- timely communication including communication of anticipated toxicities

- providing clarity of roles, responsibilities and tasks

- record sharing

- providing rapid access to acute care for advice

- providing access to training opportunities, care pathways and guidelines

- providing access to current service directories

- providing appropriate resourcing of services.

Elysia Thornton-Benko BSc, MBBS (Hons), PhD, FRACGP, Specialist General Practitioner, Sydney, NSW; Research Fellow, Behavioural Sciences Unit, Faculty of Medicine, University of New South Wales, Sydney, NSW; Kids Cancer Centre, Sydney Children's Hospital, Randwick, NSW

Richard J Cohn MBBCh, DCH, FRACP, Paediatric Haematologist Oncologist, Director of Survivorship Program, Kids Cancer Centre, Sydney Children's Hospital, Randwick, NSW; Professor, School of Women's and Children's Health, University of New South Wales, Sydney, NSW

Raymond I Chan RN, BNurs, MAppSci, PhD, FACN Director and Professor in Cancer Nursing, Caring Futures Institute, College of Nursing and Health Sciences, Flinders University, SA

Joel Rhee BSc (Med), MBBS (Hons), GCULT, PhD, FRACGP, Associate Professor of General Practice, General Practice Academic Unit, School of Medicine, Faculty of Science, Medicine and Health, University 


\section{Box 2. Case example: Supporting young cancer survivors}

Childhood, adolescent and young adult (CAYA) survivors represent a unique population with potentially six or more decades of life after treatment for cancer. ${ }^{24}$ Many survivors of CAYA cancer face numerous long-term health risks, which increase as they age. ${ }^{25}$ While survivors who experience significant multimorbidity require resource-intensive specialist clinic follow-up, for those with a low risk of late adverse sequelae, discharge to primary care is appropriate. For all survivors, encouraging engagement in primary care is important to promote holistic follow-up care, continuity of care and long-term surveillance regardless of risk stratification. ${ }^{4}$

\section{Case study}

Amy, aged 18 years, was treated for acute lymphoblastic leukaemia at the age of 11 years. She received two years of chemotherapy, which was well tolerated but resulted in significant time away from school. She experienced subsequent difficulties at school, with impact on her friendships. Her medical assessment revealed no late physical complications of therapy, but she required support for anxiety. She was transitioned for further follow-up to primary care, as she was stratified as low risk for late complications of her cancer and the therapy she received.

Amy and her family had an ongoing relationship with their general practitioner (GP), who had cared for Amy since she was a toddler. The cancer care team encouraged this relationship and flagged transition to care early so appropriate plans could be made, and to prevent interruption to engagement with the GP and instill confidence in the GP. The survivorship care plan provided to Amy and her GP contained an end-of-treatment summary with details of all treatment received, as well as recommendations for follow-up that included a yearly physical examination, yearly influenza vaccination, support as necessary for her anxiety, a surveillance echocardiogram every five years in view of her exposure to anthracyclines, and cardiac review during pregnancy.

The plan also contained advice about following a healthy lifestyle, as this has been shown to decrease the risk of late effects. Access to the long-term follow-up nurse coordinator was made available for Amy and the GP. The GP arranged a care planning meeting with Amy to agree on goals of care. During that appointment, Amy identified her priorities as completion of her studies, focus on increasing independence and need for peer support.

Together with Amy, the GP created a surveillance and support plan including regular GP reviews to ensure ongoing support. The potential for a GP Mental Health Treatment Plan was discussed, with associated referral to a psychologist, to assist Amy with managing her anxiety and learning effective coping strategies. The GP informed Amy about other allied health professionals and their potential roles, such as an occupational therapist to aid with completing studies and liaising with the education provider and to become aware of the education supports available. The GP also discussed social supports and encouraged some participation in a hobby/sport/recreation. Amy admitted that exercising helped her to feel motivated and energised while improving her mental health. Amy was aware of the importance of maintaining exercise for not only general health and wellbeing but also long term for cancer survivors generally. She was also keen to recommence playing the saxophone in a local music group and to rekindle some past friendships.

The GP informed Amy about health screenings and their timings, in addition to discussing reproductive and sexual health. Amy was also cautioned on the consumption of alcohol and was advised to abstain from ever smoking, vaping or using illicit drugs. Amy understood the importance of her survivorship plan. Amy felt confident that she could discuss issues or concerns with her GP and was aware that the long-term follow-up clinic was also available to provide further assistance if/when indicated.

of Wollongong, Wollongong, NSW; Illawarra Health and Medical Research Institute, Wollongong, NSW; General Practitioner, HammondCare Centre for Positive Ageing and Care, Hammondville, NSW David Joske MBBS, FRACP, FRCPA, FCHSM, Clinical Haematologist, Sir Charles Gairdner Hospital, Nedlands, WA; Clinical Professor of Medicine, University of Western Australia, Crawley, WA
Mahesh Iddawela MBBS, PhD, Medical Oncologist, Department of Medical Oncology, Alfred Health, Melbourne, Vic; Monash University, Melbourne, Vic Janette L Vardy BMed (Hons), FRACP, PhD, Professor of Cancer Medicine, Faculty of Medicine and Health, University of Sydney, Sydney, NSW; Concord Cancer Centre, Concord Repatriation General Hospital, Concord, NSW
Competing interests: JR is a member of the Australian Journal of General Practice Editorial Advisory Committee.

Funding: None.

Provenance and peer review: Commissioned, externally peer reviewed.

\section{Correspondence to:}

bogda.koczwara@flinders.edu.au

\section{References}

1. Markham MJ, Wachter K, Agarwal N, et al. Clinical cancer advances 2020: Annual report on progress against cancer from the American Society of Clinical Oncology. J Clin Oncol 2020;38(10):1081. doi: 10.1200/JCO.19.03141. Erratum in: J Clin Oncol 2020;38(26):3076. doi: 10.1200/JCO.20.02291.

2. Moscow JA, Fojo T, Schilsky RL. The evidence framework for precision cancer medicine. Nat Rev Clin Oncol 2018;15(3):183-92. doi: 10.1038/ nrclinonc.2017.186.

3. Jemal A, Bray F, Center MM, Ferlay J, Ward E, Forman D. Global cancer statistics. CA Cancer J Clin 2011;61(2):69-90. doi: 10.3322/caac.20107. Erratum in: CA Cancer J Clin 2011;61(2):134.

4. Cherny NI, de Vries EG, Emanuel L, et al. Words matter: Distinguishing 'Personalized medicine' and 'Biologically personalized therapeutics'. J Natl Cancer Inst 2014;106(12):dju321. doi: 10.1093/ jnci/dju321.

5. World Health Organization. Quality of care: A process for making strategic choices in health systems. Geneva, CH: WHO, 2006.

6. Institute of Medicine (US) Committee on Quality of Health Care in America. Crossing the quality chasm: A new health system for the 21st century. Washington, DC: National Academies Press, 2001.

7. Licqurish SM, Cook OY, Pattuwage LP, et al. Tools to facilitate communication during physician-patient consultations in cancer care: An overview of systematic reviews. CA Cancer J Clin 2019;69(6):497-520. doi: 10.3322/caac.21573.

8. Lawn S, Battersby M. Chronic condition management models for cancer care and survivorship. In: Koczwara B, editor. Cancer and chronic conditions: Addressing the problem of multimorbidity in cancer patients and survivors. Singapore, SG: Springer, 2016; p. 241-59.

9. Howell D, Mayer DK, Fielding R, et al. Management of cancer and health after the clinic visit: A call to action for self-management in cancer care. J Natl Cancer Inst 2021;113(5):523-31. doi: 10.1093/jnci/djaa083.

10. Barlow J, Wright C, Sheasby J, Turner A, Hainsworth J. Self-management approaches for people with chronic conditions: A review. Patient Educ Couns 2002;48(2):177-87. doi: 10.1016/ s0738-3991(02)00032-0.

11. Dineen-Griffin S, Garcia-Cardenas V, Williams K, Benrimoj SI. Helping patients help themselves: A systematic review of self-management support strategies in primary health care practice. PLoS One 2019;14(8):e0220116. doi: 10.1371/journal. pone.0220116.

12. Harris MF, Williams AM, Dennis SM, Zwar NA, Powell Davies G. Chronic disease self-management: Implementation with and within Australian general practice. Med J Aust 2008;189 Suppl 10:S17-20. doi: 10.5694/j.1326-5377.2008.tb02204.x.

13. Reynolds R, Dennis S, Hasan I, et al. A systematic review of chronic disease management interventions in primary care. BMC Fam Pract 2018;19(1):11. doi: 10.1186/s12875-017-0692-3.

14. Hoey LM, leropoli SC, White VM, Jefford M. Systematic review of peer-support programs for people with cancer. Patient Educ Couns 2008;70(3):315-37. doi: 10.1016/j.pec.2007.11.016. 
15. Ritchie CS, Kvale E, Fisch MJ. Multimorbidity: An issue of growing importance for oncologists. J Oncol Pract 2011;7(6):371-74. doi: 10.1200/ JOP.2011.000460.

16. Nekhlyudov L, Mollica MA, Jacobsen PB, Mayer DK, Shulman LN, Geiger AM. Developing a quality of cancer survivorship care framework: Implications for clinical care, research, and policy. J Natl Cancer Inst 2019;111(11):1120-30. doi: 10.1093/jnci/djz089. Erratum in: J Natl Cancer Inst 2021;113(2):217.

17. Fox $P$, Boyce $A$. Cancer health inequality persists in regional and remote Australia. Med J Aust 2014;201(8):445-46. doi: 10.5694/mja14.01217.

18. Collins IM, Burbury K, Underhill CR. Teletrials: Implementation of a new paradigm for clinical trials. Med J Aust 2020;213(6):263-65.e1. doi: $10.5694 / \mathrm{mja} 2.50741$.

19. Pearce A, Tomalin B, Kaambwa B, et al. Financial toxicity is more than costs of care: The relationship between employment and financial toxicity in long-term cancer survivors. J Cancer Surviv 2019;13(1):10-20. doi: 10.1007/s11764-018-0723-7.
20. World Health Organization. Health promotion glossary. Geneva, CH: WHO, 1998.

21. Australian Digital Inclusion Alliance. National digital inclusion roadmap. Melbourne, Vic: ADIA, 2020.

22. Kemp E, Trigg J, Beatty L, et al. Health literacy, digital health literacy and the implementation of digital health technologies in cancer care: The need for a strategic approach. Health Promot J Austr 2021;32 Suppl 1:S104-14. doi: 10.1002/ hpja.387.

23. Jefford M, Koczwara B, Emery J, Thornton-Benko E, Vardy JL. The important role of general practice in the care of cancer survivors. Aust J Gen Pract 2020;49(5):288-92. doi: 10.31128/AJGP-10-19-5133.

24. Signorelli C, Fardell JE, Wakefield CE, et al. The cost of cure: Chronic conditions in survivors of child, adolescent, and young adult cancers. In: Koczwara B, editor. Cancer and chronic conditions: Addressing the problem of multimorbidity in cancer patients and survivors. Singapore, SG: Springer, 2016; p. 371- 420.
25. Suh E, Stratton $\mathrm{KL}$, Leisenring WM, et al. Late mortality and chronic health conditions in longterm survivors of early-adolescent and young adult cancers: A retrospective cohort analysis from the Childhood Cancer Survivor Study. Lancet Oncol 2020;21(3):421-35. doi: 10.1016/S14702045(19)30800-9. 